\title{
Communication: Disorder-suppressed vibrational relaxation in vapor-deposited high-density amorphous ice
}

\author{
Shalit, Andrey ; Perakis, Fivos ; Hamm, Peter
}

\begin{abstract}
We apply two-dimensional infrared spectroscopy to differentiate between the two polyamorphous forms of glassy water - low-density (LDA) and high-density (HDA) amorphous ices, that were obtained by slow vapor deposition at 80 and $11 \mathrm{~K}$ respectively. Both the vibrational lifetime and the bandwidth of the 1-2 transition of the isolated OD stretch vibration of HDO in $\mathrm{H} 2 \mathrm{O}$ exhibit characteristic differences when comparing hexagonal (Ih), LDA and HDA ices, which we attribute the different local structures - in particular the presence of interstitial waters in HDA ice - that cause different delocalization lengths of intermolecular phonon degrees of freedom. Moreover, temperature dependent measurements show that the vibrational lifetime closely follows the structural transition between HDA and LDA phases.
\end{abstract}

DOI: https://doi.org/10.1063/1.4871476

Posted at the Zurich Open Repository and Archive, University of Zurich

ZORA URL: https://doi.org/10.5167/uzh-99224

Journal Article

Accepted Version

Originally published at:

Shalit, Andrey; Perakis, Fivos; Hamm, Peter (2014). Communication: Disorder-suppressed vibrational relaxation in vapor-deposited high-density amorphous ice. Journal of Chemical Physics, 140(15):151102. DOI: https://doi.org/10.1063/1.4871476 


\title{
Disorder-suppressed vibrational relaxation in vapor-deposited high-density amorphous ice
}

\author{
Andrey Shalit, Fivos Perakis and Peter Hamm \\ Department of Chemistry, University of Zurich, Winterthurerstrasse 190, \\ CH-8057Zurich, Switzerland, phamm@pci.uzh.ch
}

(Dated: April 10, 2014)

\begin{abstract}
We apply two-dimensional infrared spectroscopy to differentiate between the two polyamorphous forms of glassy water - low-density (LDA) and high-density (HDA) amorphous ices, that were obtained by slow vapor deposition at 80 and $11 \mathrm{~K}$ respectively. Both the vibrational lifetime and the bandwidth of the 1-2 transition of the isolated OD stretch vibration of HDO in $\mathrm{H}_{2} \mathrm{O}$ exhibit characteristic differences when comparing hexagonal (Ih), LDA and HDA ices, which we attribute the different local structures - in particular the presence of interstitial waters in HDA ice - that cause different delocalization lengths of intermolecular phonon degrees of freedom. Moreover, temperature dependent measurements show that the vibrational lifetime closely follows the structural transition between HDA and LDA phases.
\end{abstract}

Along with a variety of crystalline ice polymorphs (at least 15 phases are known today [1-3]), water can also exists in more than one amorphous form in its glassy state [4-6]. There are two main types of amorphous ice: low-density amorphous (LDA) and high-density amorphous (HDA) ice. LDA was first observed more than 70 years ago [7] by vapor deposition onto a cold plate, while HDA was first obtained by Mishima et al. through the compression of the hexagonal ice in 1984 [8]. Recently, an additional distinct structural state of amorphous ice, very high-density amorphous ice (VHDA), was acquired upon isobaric heating of HDA under pressure [9]. This polyamorphism is believed to be a manifestation of the multi-component structural aggregates in liquid water which are responsible for the non-monotonous behavior of its various thermodynamic and dynamic properties $[10,11]$. In the context of the liquid-liquid phase transition hypothesis proposed by Poole et al. [12], LDA and HDA are considered to be the solid-state structural analogs of the low and the high density liquid (LDL and HDL) - two distinct liquid phases below the hypothesized second critical point.

Various experimental techniques including Raman [13, 14], IR [15, 16] and NMR [17] spectroscopy as well as $\mathrm{X}$-ray $[18,19]$ and neutron diffraction $[20-22]$ have been applied to determine the structural properties of LDA and HDA. In particular with the help of neutron diffraction together with isotope substitution, it has been established that additional water molecules populate an interstitial position in HDA between the first and the second coordination shell, which results in its higher density.

Recently, we investigated isotope diluted LDA by ultrafast two-dimensional infrared (2D IR) spectroscopy [23]. 2D IR spectroscopy [24-28] monitors the local structure and dynamics of the hydrogen bonds in water through the frequency of the hydroxyl stretch vibration [29-32]. We demonstrated that the high structural disorder of LDA on the one hand, and the complete lack of spectral diffusion on the other hand, allows one to observe how various spectral properties of the hydroxyl stretch vibration varies with hydrogen bond strength to the water molecules in the first coordination shell.

In this contribution, we address the structural differences of the two amorphous forms of ice with the help of 2D IR spectroscopy. By comparing 2D IR spectra of hexagonal (Ih), LDA and HDA ice, we will demonstrate how vibrational relaxation, lineshapes and diagonal anharmonicity are correlated with structural motifs, revealing a signature of the interstitial water molecule in HDA.

As mentioned above, HDA ice is usually obtained by pressurizing ice Ih at low temperatures [8]. In this work, we use a different preparation method, in which HDA is produced by slow vapor deposition onto a substrate at $11 \mathrm{~K}$. In this way, an ultrathin film of ice is obtained which is a crucial requirement for transmission spectroscopes such as $2 \mathrm{D}$ IR. It is important to stress that vapor-deposited form of HDA used in this work can not be considered as fully analogous to the HDA obtained through pressure-induced amorphisation. As expected for glasses in general, different preparation procedures leads to variation in the resulting state. The transition temperature to LDA provides probably the most striking evidence regarding the difference between both forms: pressure-induced HDA relaxes to LDA at $130 \mathrm{~K}$ [5] while vapor-deposited HDA shows a significatively lower transition temperature of $38 \mathrm{~K}$ [33]. Nevertheless, on basis of electron diffraction it has been shown that both versions of HDA are very similar in terms of density and structure [34]. Most importantly, both vapor-deposited and pressure-induced HDA forms posses an additional water molecule at the "interstitial" site, allowing one to draw a reasonable analogy between both forms with regard to the effect of structural disorder on the vibrational dynamics, which is the topic of this paper. From this point on, the term "HDA" will be preserved for vapor-deposited HDA unless stated otherwise.

We start with comparing IR absorption spectra of ice Ih (Fig. 1, green dotted line), LDA (red dashed line) and HDA (blue solid line). Throughout this paper, we will investigate the OD-stretch vibration of $10 \%$ HDO in $\mathrm{H}_{2} \mathrm{O}$. Isotope diluted HDA $\left(\rho=1.17 \mathrm{~g} / \mathrm{cm}^{3}\right)$, LDA $\left(\rho=0.94 \mathrm{~g} / \mathrm{cm}^{3}\right)$ and $\mathrm{Ih}\left(\rho=0.94 \mathrm{~g} / \mathrm{cm}^{3}\right)$ ices were pro- 


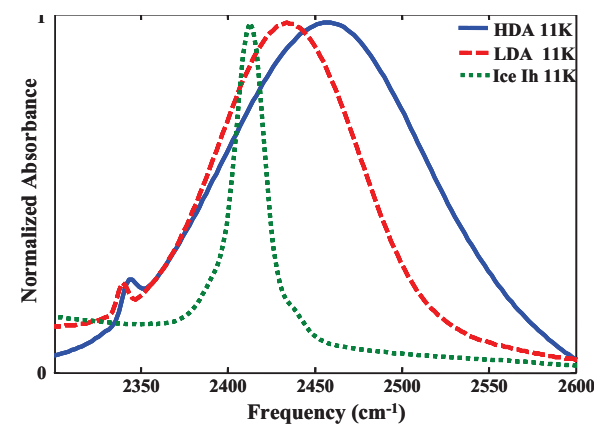

FIG. 1: Normalized absorption spectra of $\mathrm{HDO}$ in $\mathrm{H}_{2} \mathrm{O}$ spectrum in ice Ih (green dotted line), LDA (red dashed line), HDA ice (blue solid line). The small peak below $2350 \mathrm{~cm}^{-1}$ originates from trace amounts of $\mathrm{CO}_{2}$.

duced by slow vapor deposition with rate of less then $0.1 \mu \mathrm{m} / \mathrm{min}$ onto a cold $\mathrm{CaF}_{2}$ window held at 11,80 and $150 \mathrm{~K}$, respectively, in a closed-cycle He cryostat. The ice Ih and LDA samples were subsequently cooled down to the desired temperatures. The $\mathrm{HDO} / \mathrm{H}_{2} \mathrm{O}$ mixture was degassed before deposition by a freeze-pumpthaw procedure, and the thickness of the ice layer was monitored in-situ by monitoring the transmission of the probe pulse during deposition. The deposition process was stopped once an optical density of 0.15 was reached for all three samples, which corresponds to the thickest layer $(\approx 0.5 \mu \mathrm{m})$ for which HDA still remains stable at $11 \mathrm{~K}$. All samples show reproducible spectroscopic properties under our experimental conditions.

Fig. 1 shows that both amorphous phases exhibit broader absorption bands than ice Ih due to the larger structural disorder in the glassy state. The absorption band in HDA is shifted a bit to higher frequencies compared to LDA (peaking at $2460 \mathrm{~cm}^{-1}$ vs. $2435 \mathrm{~cm}^{-1}$, respectively), which implies that albeit its higher density, on average, the hydrogen bond strength in HDA is weaker. The average $\mathrm{O} \cdots \mathrm{O}$ distances of $2.77 \AA$ and $2.82 \AA$ for LDA and pressure-induced HDA, respectively, were previously reported $[17,18]$. This counter-intuitive density/hydrogen bond strength correlation is usually explained by the presence of additional interstitial water molecules found between the first and the second coordination shell in HDA, which have indeed been observed in the oxygen-oxygen radial distribution function derived from X-ray and neutron scattering experiments [21]. These interstitial water molecules force water molecules involved in the tetrahedral network to move slightly apart, thus increasing the average $\mathrm{O} \cdots \mathrm{O}$ distance.

2D IR spectra were recorded in pump-probe geometry using an interferometer to generate two collinear phaselocked pump-pulses separated by coherence time $t_{1}$ [35]. A second delay stage shifted the two pump pulses with respect to the probe pulse, referred to as population time $t_{2}$. Unwanted interference effects resulting from scattering from the ice samples are suppressed with help of a wobbling Brewster window [36]. The probe beam was

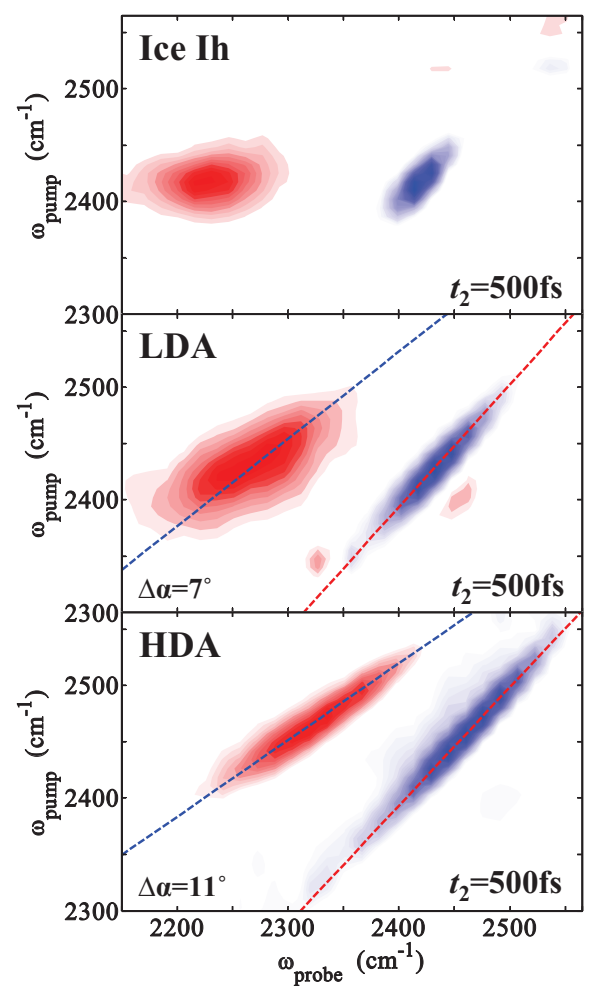

FIG. 2: Normalized anisotropic $\left(S_{\mathrm{ZZZZ}}-S_{\mathrm{ZZYY}}\right) 2 \mathrm{D}$ IR spectra of the OD stretch vibration of ice Ih, LDA and HDA (from top to bottom) at $11 \mathrm{~K}$ for population times $t_{2}=500 \mathrm{fs}$. We depict the pump frequency axis $\left(\omega_{\text {pump }}\right)$ vertically and the probe frequency axis $\left(\omega_{\text {probe }}\right)$ horizontally. Linear fit for minima (dashed red line) and maxima (dashed blue line) along the transition lobes are shown for LDA and HDA ices with the corresponding slope differences $\Delta \alpha$.

sent through a spectrograph onto an array MCT detector for frequency-resolved detection. We scanned $t_{1}=$ $(-300,1000)$ fs and recorded spectra for various population times $t_{2}$ delays up to $5 \mathrm{ps}$.

Figure 2 shows normalized 2D IR spectra of the OD stretch vibration of ice Ih, LDA and HDA for a population time $t_{2}=500$ fs at $11 \mathrm{~K}$. Anisotropic responses are shown, constructed from two separate 2D IR measurements with two orthogonal pump polarizations $\left(S_{\mathrm{ZZZZ}}-S_{\mathrm{ZZYY}}\right)$. This procedure separates vibrational (anisotropic) from thermal (isotropic) signals by exploiting the lack of orientation motion in the ice samples [23]. The on-diagonal (blue) peaks are associated with bleach and stimulated emission between ground and first excited state (0-1 transition), while the oppositely signed off-diagonal peaks (red) are due to the anharmonically shifted absorption from the first to the second excited state (1-2 transition). For all three samples, the lineshapes of $0-1$ transition are consistent in terms of bandwidth and position with the corresponding absorption spectra (Fig. 1). LDA and HDA show a diagonally elongated narrow $0-1$ peak, reflecting the significant amount of inhomogeneous broadening in the glassy state. Very 


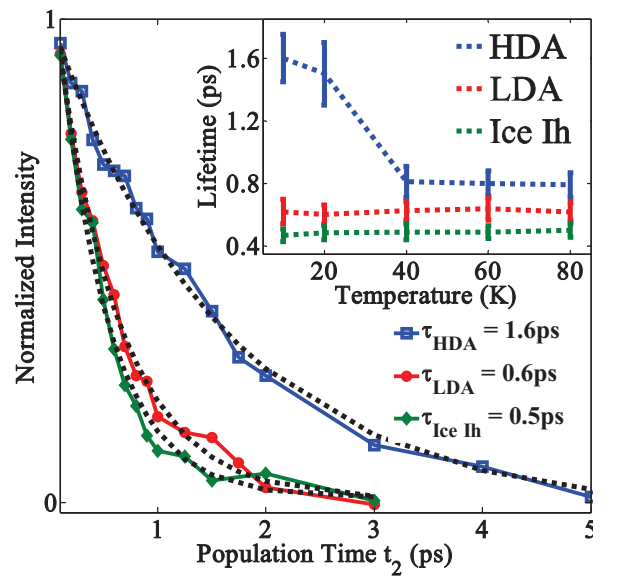

FIG. 3: Decay of the 1-2 signal for HDA (blue), LDA(red) and ice Ih (green) as function of population time $t_{2}$, in all cases taken at $\left(\omega_{\text {pump }}=2412 \mathrm{~cm}^{-1}, \omega_{\text {probe }}=2210 \mathrm{~cm}^{-1}\right)$, which corresponds to the peak for ice Ih. The inset shows decay constants of all three ices as function of temperature with consistent color code.

recently, this observation was also confirmed with help of molecular dynamic simulations [37].

A small inhomogeneous broadening, possibly due to proton disorder is also observed for ice Ih [38, 39]. More revealing is the 1-2 transition, for which significant variations in the lineshapes are found for the three different samples. Both amorphous ices exhibit a dependence of the anharmonic shift as well as of the linewidth of the 12 transition with the excitation frequency, while the 1-2 lobe remains unresolved in ice Ih due to the narrower frequency distribution. To quantify the anharmonic shifts for the amorphous samples the maxima (for 1-2 transition) and minima (for $0-1$ transition) of the spectral slices along the $\omega_{\text {pump }}$ axis were linearly fitted. The difference between the slopes of $0-1$ and 1-2 transition reflects the stronger dependence of anharmonicity on the excitation frequency in $\operatorname{HDA}\left(\Delta \alpha=11^{\circ}\right)$ vs. $\operatorname{LDA}\left(\Delta \alpha=7^{\circ}\right)$.

Due to the lack of spectral diffusion, no significant change of lineshape is observed as a function population time $t_{2}$ in any of the samples, but the overall intensity decreases due to vibrational relaxation. The decay of $1-2$ transition is considered to be a reliable measure of vibrational lifetime as it directly reflects the population of the first excited state. The intensity of the 1-2 band is shown in Fig. 3 for HDA (blue), LDA (red) and Ih (green). Dashed lines in Fig. 3 correspond to single exponential fits (within signal to noise there is no indication of nonexponential behavior), which reveal a relaxation times in HDA $(1.6 \pm 0.2 \mathrm{ps})$ that is significantly slower than in LDA $(0.6 \pm 0.05 \mathrm{ps})$ and $\mathrm{Ih}(0.5 \pm 0.05 \mathrm{ps})$. Note that these decay times are obtained at the same frequency positions in all three cases (i.e., $\omega_{\text {pump }}=2412 \mathrm{~cm}^{-1}$ and $\omega_{\text {probe }}=2210 \mathrm{~cm}^{-1}$, which corresponds to the peak of the 1-2 lobe in ice Ih).

The comparison of the vibrational relaxation for the

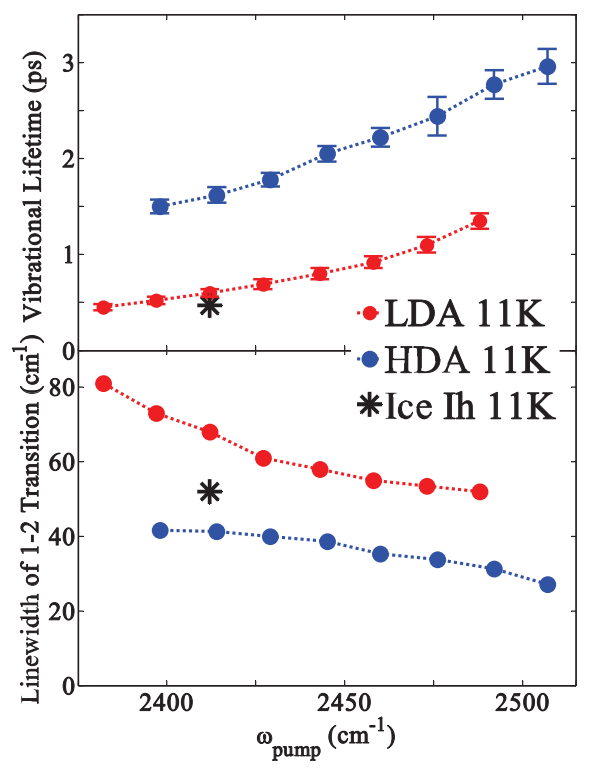

FIG. 4: (a) Vibrational lifetimes and (b) linewidth of 1-2 transition and as function of excitation frequency $\omega_{\text {pump }}$ for LDA (red) and HDA (blue). The black asterisks in both panels indicate the corresponding values for ice Ih.

subensembles of molecules absorbing at the same frequency is made in attempt to isolate, at least partially, the effect of various hydrogen bond environments on the relaxation process. The vibrational frequency of the hydroxyl stretch $(\omega)$ correlates not only with the hydrogen bond length but also with the hydrogen bond angle [40]. Thus considering molecules at the same $\omega$ does not necessarily guarantee a similarity in local hydrogen-bond arrangement (especially when ordered Ih is compared to disordered HDA). Nevertheless in essence the relationship between $\omega$ and hydrogen bond strength (which depends on both length and angle) is still considered to be valid. Comparable hydrogen bond strengths (defined as just discussed) in the various forms of ice do reveal significantly different relaxation times. Vibrational relaxation of the OD stretch vibration is believed to proceed through the HDO bending mode of the molecule itself [41-43], or the $\mathrm{H}_{2} \mathrm{O}$ bending mode of neighboring water molecules [44]; in any case, it is mediated by phonon degrees of freedom. Thus, longer range structural properties and hence more delocalized phonons must play a significant role in the relaxation process.

Due to the excessive inhomogeneous broadening and static environment of the amorphous ices, using 2D IR spectroscopy we can also measure the vibrational lifetimes as function of the excitation frequency (Fig. 4a), thus exploiting the effect of the various hydrogen bond environment on the relaxation rates. For both LDA (red) and HDA (blue), the relaxation rates correlate with vibrational frequency and hence with hydrogen bond strength in the same manner, that is, the stronger the hydrogen bond, the faster is the relaxation. The relax- 
ation rates vary by about a factor 2 throughout the accessible frequency range, and the observation we already made before, namely that vibrational relaxation in LDA is significantly faster than in HDA at a given frequency position, remains valid everywhere.

Another observable is the linewidth of the 1-2 transition, which is quite different in LDA versus HDA (Fig. 2). Figure $4 \mathrm{~b}$ shows that also this linewidth has a characteristic pump frequency dependence, and that the 1-2 transition is narrower in HDA as compared to LDA in the entire excitation range. An asymmetry of the linewidth of $0-1$ versus $1-2$ transitions has been observed for liquid water $[45,46]$ and much more prominently for ice Ih [47-49], and has been attributed to a specific coupling of the OD stretch vibration to phonon degrees of freedom. As for the vibrational relaxation time, we find that this coupling is different for LDA versus HDA for a given strength of the local hydrogen bond to the OD group, so again we conclude that these phonons must be of more delocalized nature than just the first coordination shell.

Finally, we show in Fig. 3 (inset) the vibrational lifetimes for all three forms of ice as a function of temperature, again at all the same frequency positions. Neither ice Ih (green) nor LDA (red) exhibit any significant temperature dependence. The same trend has been observed before for ice Ih and has been explained by the lack of a temperature dependence of hydrogen bond strength measured via the OD absorption frequency [50]. Also the absorption spectra of LDA show hardly any temperature dependence of the peak frequency (see Figure S1 in Supplementary Material [54]). The vibrational lifetime in HDA (blue), in contrast, decreases form 1.6 ps down to 0.8 ps between $20 \mathrm{~K}$ and $40 \mathrm{~K}$ in a step-like manner with no significant evolution further on. Such a jump suggests a major structural rearrangement in HDA in this temperature range. Indeed previous studies have shown that vapor deposited HDA undergoes a transformation to LDA around $38 \mathrm{~K}$ [33]. It is an irreversible transition, so once LDA is formed, the spectroscopic properties remain constant when decreasing temperature again below the transition temperature (see Figure S1).

It is interesting to note that the vibrational lifetime of this phase-transformed LDA remains $\approx 30 \%$ larger than of LDA produced by direct deposition at $80 \mathrm{~K}$. Thus, even though both of these samples are classified as LDA ices, small structural differences must still exist that depend on the history of the preparation protocol, not surprising for a glassy state [51-53]. The vibrational relaxation rate thus appears to be a rather sensitive probe of such minor structural differences.

A qualitatively similar step-like temperature dependence of the vibrational lifetimes has been observed for the phase transition from liquid water to ice Ih [50]. As in our case, the transition from a highly disorder phase to a more ordered phase manifests in a significant decrease of the vibrational lifetime.

In conclusion, we demonstrated and analyzed the differences of ice Ih, LDA and HDA in terms of the spectro- scopic information accessible from ultrafast 2D IR spectroscopy. We systematically varied the long range structural order from very ordered in ice Ih through LDA to the highly disordered HDA phase. From neutron diffraction it is known that all three phases are quite similar with respect to the local structure of the first coordination shell [21]. This is essentially what the absorption frequency in the IR spectrum is sensitive to - the strength of the hydrogen bond of a hydroxyl group to the directly hydrogen bonded water molecule in the first coordination shell. However, 2D IR spectroscopy can provide more information; for the purpose of the present discussion in particular about the vibrational lifetime, the linewidth of the 1-2 transition and diagonal anharmonicity. The first two are rather unspecific measures of the coupling of the hydroxyl stretch vibration to phonon degrees of freedom. While there is a correlation of hydrogen bond strength and this coupling (Fig. 4), both these measures reveal that the coupling is weaker in HDA as compared to the more ordered LDA phase or ice Ih. It is interesting to note that LDA and ice Ih appear to be essentially the same in terms of vibrational relaxation (Fig. 4a), but not in terms of the linewidth of the 1-2 transition, for which ice Ih lies between LDA and HDA (Fig. 4b). With regard to the diagonal anharmonicity, both amorphous ices behave in a consistent way, i.e., stronger hydrogen bonds induce larger anharmonic shifts. However the smaller values of anharmonic shifts observed for LDA vs. HDA cannot be explained with help of a one-dimensional OD stretch potential, suggesting that couplings to other degrees of freedom must also be considered. In any case, we consider the slower vibrational lifetime and the narrower line width of the 1-2 transition to be a signature of larger structural disorder in HDA ice due to the presence of interstitial water molecules. Given the fact that pressureinduced and vapor-deposited HDA ices are structurally similar [34], qualitatively the same behavior is expected also for pressure-induced HDA.

As a speculative explanation for the differences in LDA versus HDA we propose that it might be the larger delocalization length of phonon degrees of freedom in an more ordered system that leads to an effectively higher density of phonon states with spatial overlap with the OD group. Alternatively, it may also originate from the significant difference in the local structure. In particular, the anharmonic coupling between OD stretch vibration and hydrogen bond will depended on the angle, presumably strongly decreasing with increasing angle, resulting in slower vibrational relaxation for a strained hydrogen bond configuration. It would be interesting to address those ideas with the help of mixed-quantum/classical simulations.

Acknowledgement: We thank Jan Helbing for significant help in the lab. The work has been supported by the Swiss National Science Foundation (SNF) in a National Center of Competence and Research (NCCR) MUST as well as a EU cofund IFP-MUST. 
[1] E. A. Zheligovskaya and G. G. Malenkov, Russ. Chem. Rev. 75, 57 (2006).

[2] C. G. Salzmann, P. G. Radaelli, B. Slater, and J. L. Finney, Phys. Chem. Chem. Phys. 13, 18468 (2011).

[3] T. Bartels-Rausch, V. Bergeron, J. H. E. Cartwright, R. Escribano, J. L. Finney, H. Grothe, P. J. Gutiérrez, J. Haapala, W. F. Kuhs, J. B. C. Pettersson, et al., Rev. Mod. Phys. 84, 885 (2012).

[4] C. A. Tulk, C. J. Benmore, J. Urquidi, D. D. Klug, J. Neuefeind, B. Tomberli, and P. A. Egelstaff, Science 297, 1320 (2002).

[5] T. Loerting and N. Giovambattista, J. Phys.: Condens. Matter 18, R919 (2006).

[6] T. Loerting, K. Winkel, M. Seidl, M. Bauer, C. Mitterdorfer, P. H. Handle, C. G. Salzmann, E. Mayer, J. L. Finney, and D. T. Bowron, Phys. Chem. Chem. Phys. 13, 8783 (2011).

[7] E. F. Burton and W. F. Oliver, Proc. R. Soc. Lond. As 153, 166 (1935).

[8] O. Mishima, L. D. Calvert, and E. Whalley, Nature 310, 393 (1984).

[9] T. Loerting, C. Salzmann, I. Kohl, E. Mayer, and A. Hallbrucker, Phys. Chem. Chem. Phys. 3, 5355 (2001).

[10] I. Brovchenko and A. Oleinikova, ChemPhysChem 9, 2660 (2008).

[11] H. E. Stanley, P. Kumar, G. Franzese, L. Xu, Z. Yan, M. G. Mazza, S. V. Buldyrev, S. H. Chen, and F. Mallamace, European Physical Journal-Special Topics 161, 1 (2008).

[12] P. H. Poole, F. Sciortino, U. Essmann, and H. E. Stanley, Nature 360, 324 (1992).

[13] D. D. Klug, O. Mishima, and E. Whalley, J. Chem. Phys. 86, 5323 (1987)

[14] Y. Suzuki, Y. Takasaki, Y. Tominaga, and O. Mishima, Chem. Phys. Lett. 319, 81 (2000).

[15] T. C. Sivakumar, D. Schuh, M. G. Sceats, and S. A. Rice, Chem. Phys. Lett. 48, 212 (1977).

[16] M. S. Bergren, D. Schuh, M. G. Sceats, and S. A. Rice, J. Chem. Phys. 69, 3477 (1978).

[17] J. A. Ripmeester, C. I. Ratcliffe, and D. D. Klug, J. Chem. Phys. 96, 8503 (1992).

[18] A. Bizid, L. Bosio, A. Defrain, and M. Oumezzine, J. Chem. Phys. 87, 2225 (1987).

[19] T. Loerting, W. Schustereder, K. Winkel, C. G. Salzmann, I. Kohl, and E. Mayer, Phys. Rev. Lett. 96, 025702 (2006).

[20] J. L. Finney, D. T. Bowron, A. K. Soper, T. Loerting, E. Mayer, and A. Hallbrucker, Phys. Rev. Lett 89, 205503 (2002).

[21] J. L. Finney, A. Hallbrucker, I. Kohl, A. K. Soper, and D. T. Bowron, Phys. Rev. Lett 88, 225503 (2002).

[22] K. Winkel, D. T. Bowron, T. Loerting, E. Mayer, and J. L. Finney, J. Chem. Phys. 130, 204502 (2009).

[23] A. Shalit, F. Perakis, and P. Hamm, J. Phys. Chem. B. 117, 15512 (2013)

[24] J. B. Asbury, T. Steinel, K. Kwak, S. A. Corcelli, C. P. Lawrence, J. L. Skinner, and M. D. Fayer, J. Chem. Phys. 121, 12431 (2004).

[25] J. D. Eaves, J. J. Loparo, C. J. Fecko, S. T. Roberts, A. Tokmakoff, and P. L. Geissler, Proc. Natl. Acad. Sci. USA 102, 13019 (2005).
[26] S. Yeremenko, M. S. Pshenichnikov, and D. A. Wiersma, Chem. Phys. Lett. 369, 107 (2003).

[27] M. L. Cowan, B. D. Bruner, N. Huse, J. R. Dwyer, B. Chugh, E. T. J. Nibbering, T. Elsaesser, and R. J. D. Miller, Nature 434, 199 (2005).

[28] P. Hamm and M. T. Zanni, Concepts and Methods of $2 D$ Infrared Spectrsocopy (Cambridge University Press, Cambridge, 2011).

[29] G. M. Gale, G. Gallot, F. Hache, N. Lascoux, S. Bratos, and J. C. Leicknam, Phys. Rev. Lett. 82, 1068 (1999).

[30] S. A. Corcelli, C. P. Lawrence, and J. L. Skinner, J. Chem. Phys. 120, 8107 (2004).

[31] K. B. Moller, R. Rey, and J. T. Hynes, J. Phys. Chem. A 108, 1275 (2004).

[32] J. D. Smith, C. D. Cappa, K. R. Wilson, R. C. Cohen, P. L. Geissler, and R. J. Saykally, Proc. Natl. Acad. Sci. USA 102, 14171 (2005).

[33] P. Jenniskens and D. Blake, Science 265, 753 (1994).

[34] P. Jenniskens, D. F. Blake, M. A. Wilson, and A. Pohorille, Astrophys. J. 455, 389 (1995).

[35] J. Helbing and P. Hamm, J. Opt. Soc. Am. B 28, 171 (2011).

[36] R. Bloem, S. Garrett-Roe, H. Strzalka, P. Hamm, and P. Donaldson, Opt. Express 18, 27067 (2010).

[37] C. J. Tainter, L. Shi, and J. L. Skinner, J. Chem. Phys. 140, 134503 (2014).

[38] P. V. Hobbs, Ice Physics (Oxford University Press, Oxford, 1974)

[39] L. Shi and J. L. Skinner, J. Phys. Chem. B 117, 15536 (2013).

[40] R. Rey, K. Møller, and J. T. Hynes, J. Phys. Chem. A 106, 11993 (2002)

[41] J. C. Dek, S. T. Rhea, L. K. Iwaki, and D. D. Dlott, J. Phys. Chem. A 104, 4866 (2000).

[42] Z. Wang, A. Pakoulev, Y. Pang, and D. D. Dlott, J. Phys. Chem. A 108, 9054 (2004).

[43] R. Rey, F. Ingrosso, T. Elsaesser, and J. T. Hynes, J. Phys. Chem. A 113, 8949 (2009).

[44] W. J. Smit and H. J. Bakker, J. Chem. Phys. 139, 204504 (2013).

[45] R. Laenen, K. Simeonidis, and A. Laubereau, J. Phys. Chem. B 106, 408 (2002).

[46] H. J. Bakker and H. K. Nienhuys, Science 297, 587 (2002).

[47] A. M. Dokter and H. J. Bakker, J. Chem. Phys. 128, $024502(2008)$.

[48] F. Perakis, S. Widmer, and P. Hamm, J. Chem. Phys. 134, 204505 (2011).

[49] F. Perakis, J. Borek, and P. Hamm, J. Chem. Phys. 139, 014501 (2013).

[50] S. Woutersen, U. Emmerichs, H. K. Nienhuys, and H. J. Bakker, Phys. Rev. Lett 81, 1106 (1998).

[51] C. A. Tulk, D. D. Klug, R. Branderhorst, P. Sharpe, and J. A. Ripmeester, J. Chem. Phys. 109, 8478 (1998).

[52] D. D. Klug, C. A. Tulk, E. C. Svensson, and C. K. Loong, Phys. Rev. Lett 83, 2584 (1999).

[53] J. J. Shephard, J. S. O. Evans, and C. G. Salzmann, J. Phys. Chem. Lett. 4, 3672 (2013).

[54] See Supplementary Material Document No ... for temperture dependent IR spectra of HDA and LDA. 